\title{
Water surface topology of supercritical junction flow
}

\author{
Gašper Rak $^{1 *}$, Marko Hočevar ${ }^{2}$, Franci Steinman $^{1}$ \\ ${ }^{1}$ Chair of Fluid Mechanics, Faculty for Civil and Geodetic Engineering, University of Ljubljana, Jamova 2, 1000 Ljubljana, Slovenia. \\ ${ }^{2}$ Laboratory for Water Turbine Machinery, Faculty of Mechanical Engineering, University of Ljubljana, Ljubljana, Slovenia. \\ *Corresponding author. Tel.: +386 1 4254052. E-mail: gasper.rak@fgg.uni-lj.si
}

\begin{abstract}
The complexity of flow conditions at junctions amplifies significantly with supercritical flow. It is a pronounced three-dimensional two-phased flow phenomenon, where standing waves with non-stationary water surface are formed. To analyse the hydrodynamic conditions at an asymmetric right-angled junction with incoming supercritical flows at Froude numbers between 2 and 12, an experimental approach was used. For a phenomenological determination of the relations between the integral parameters of incoming flows and the characteristics of standing waves at the junction area, water surface topographies for 168 scenarios at the junction were measured using non-intrusive measurement techniques. The new, phenomenologically derived equations allow for determination of location, height and extent of the main standing waves at the junction. Research results give important information on the processes and their magnitude for engineering applications.
\end{abstract}

Keywords: Junction; Hydraulic experimentation; Standing wave; Supercritical flow; Flow patterns.

\section{INTRODUCTION}

At junctions of two or more open channels, especially under supercritical incoming flows (Froude number Fr is greater than 1; Fr gives the ratio of the flow inertia to the external gravity field $-F r=\frac{v}{\sqrt{g l}}$, a turbulent three-dimensional flow is generated. Due to high flow velocities and turbulent shear stress the two-phase flow with strong water surface dynamics along the shear layer between both incoming flows also occurs. Junctions are commonly found elements in natural hydrographic networks and hydraulic structures, and a knowledge of flow conditions at a junction is important for both the planning of developments at the junction itself, of riparian and potential bridging structures, as well as for the understanding of accompanying processes, such as sediment transport dynamics and variations in bathymetry. Despite the high quality of modern 3D numerical models, their use in precise simulations of flow conditions at junctions is only possible with adequately set initial and boundary conditions, acquired by field or laboratory measurements. To analyse the characteristics of the flow with high Froude numbers at the junction the experimental approach was selected. Experiments were conducted with an experimental apparatus of a T-shaped junction. At the junction of two supercritical incoming flows typical water flow patterns or standing waves form due to high, predominantly vertical dynamics of the water surface. The topography of these standing waves was recorded using a non-intrusive LIDAR measurement method with high spatial and temporal resolution allowing for the analysis of its main characteristics. Based on the analysis of flow patterns at the junction for a large set of scenarios, the phenomenologically derived equations between the integral parameters of incoming flows and characteristics of standing waves were developed. These equations describe the characteristics of standing waves at right-angled junctions, which, by calculating the magnitude and height of wave peaks, allows for the proper planning of developments at such junctions (designing junction structures on sewer systems, freeboard and hydraulic structures (e.g. culverts) at torrent junctions, junctions of road drainage channels etc.).

\section{PREVIOUS RESEARCH}

Research of hydraulic conditions at the junction began with an experiment by E. H. Taylor in 1944, yielding a onedimensional model for determining the ratio of depths up- and downstream of the junction based on the law of conservation of momentum for subcritical flow (Froude number Fr is smaller than 1). Both sub- and supercritical flows were observed by Bowers, (1950); Behlke and Pritchett, (1966); Greated, (1968); Best and Reid, (1984); Hager, (1989a); Hager, (1989b); Mignot et al., (2008); Pinto Coelho, (2015). Research of hydraulic conditions at the junction also included different Froude numbers in the up- and downstream sections, different junction angles, including symmetric and asymmetric junctions. Velocity fields, water mixing zones and flow conditions in junctions with and without bed discordance of the tributary channel were observed. Christodoulou (1993) analysed conditions for formation of hydraulic jump at junctions with angles of $17^{\circ}$ and $90^{\circ}$ for weak supercritical flow in the main channel $(\mathrm{Fr} \leq 2)$ and subcritical flow in the tributary channel. For supercritical approach flow Pfister and Gisonni (2014) and Saldarriaga et al. (2017) investigated flow conditions at junction manholes on circular conduits.

Limited research has been done so far in the field of hydrodynamic conditions and water surface topography at an asymmetric junction with incoming supercritical flows at higher Froude numbers $(\mathrm{Fr} \geq 2)$. The analyses of hydrodynamic conditions were mostly focused on the subcritical flow regime and supercritical flow regime at lower Froude numbers $(\mathrm{Fr} \leq 2)$ for different types of junction, but water surface topology of supercritical junction flow was previously studied only by Schwalt and Hager (1995). Their experimental research included several different scenarios, all with supercritical flow of high Froude number $(\mathrm{Fr} \leq 12)$ and with horizontal bottom throughout. They provided equations for the description of unobstructed junction flow (main dimensions of standing waves). However, their research focused on junctions with angles of $30^{\circ}$ and $60^{\circ}$, so that their equations do not apply for junctions with junction angles greater than $60^{\circ}$. 


\section{EXPERIMENTAL SET-UP AND MEASURING TECHNIQUE \\ Model set-up}

The junction of two supercritical incoming flows was investigated at the hydraulic laboratory of the Faculty of Civil and Geodetic Engineering, the University of Ljubljana. The asymmetric junction with a $90^{\circ}$ angle between main channel and tributary channel ( $T$-junction) was considered. The model is shown in Figure 1, where the main parts are marked with numbers: 1 - pipeline system equipped with valves and flowmeter; 2 - pressure vessels with adjustable height of the openings; 3 main channel; 4 - tributary channel; 5 - rails with carrier for mounting and precise positioning of measurement devices; 6 laser scanner; 7 - free overflow at the end of the model. The lengths of main and tributary channels upstream of the junction were $1 \mathrm{~m}$ to ensure equal upstream conditions while the length of main channel downstream of the junction was $5 \mathrm{~m}$. The length of channels upstream of the junction and roughness of the glass wall were sufficient for the development of supercritical steady uniform flow at the inflow. The width of all channels was constant at $0.5 \mathrm{~m}$. A horizontal bottom of all sections was maintained throughout the entire model. Glass plates were used for construction of the experimental apparatus: sharp edges, minimising the effect of the boundary layer and improving visibility though the model walls. Using large-size glass plates and rationally optimised construction also enabled the minimisation of the number and effect of joints in the entire model and thus disturbance of the flow by streamline separation.

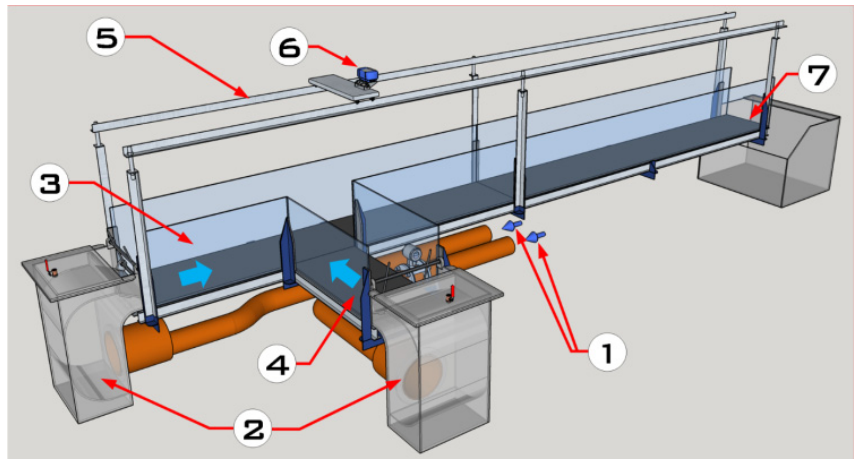

Fig. 1. Experimental set-up.

The inflows into the main and tributary channels from the reservoir were separated from each other with two pipelines. For independent settings of discharge to each inflow, both pipelines were equipped with a valve and $\mathrm{ABB}$ FlowMaster electromagnetic flowmeters with a nominal diameter of 150 $\mathrm{mm}$. The inflows with desired characteristics (flow depth, Froude number) were provided with pressure vessels, where flaps at both pressure vessels allowed for the adjustment of the opening to $0.1 \mathrm{~mm}$. Outflow from the model was provided with free overflow at the end of the main channel. Froude numbers at low depths $(1 \mathrm{~cm})$ exceeded 20 while higher depths $(5 \mathrm{~cm})$ resulted in larger discharge and due to the limited capacity of the pump achievable Froude numbers were below 4. Characteristics of the incoming flows provided in the model are presented in Table 1.

The experimental apparatus was equipped with a frame structure for mounting and precise positioning of measuring devices and accessories (Fig. 1). The frame structure with rails and a rail carrier was separately mounted on a rigid metal supporting construction to suppress vibrations. Separate mounting
Table 1. Maximum values of discharge and Froude numbers at different depths of the incoming flows.

\begin{tabular}{|c|c|c|c|}
\hline $\begin{array}{c}\text { Water depth of } \\
\text { inflows } \\
(\mathrm{mm})\end{array}$ & $\begin{array}{c}\text { Max. discharge } \\
\text { of inflows } \\
\left(\mathrm{m}^{3} / \mathrm{s}\right)\end{array}$ & $\begin{array}{c}\text { Max. velocities of } \\
\text { the inflows } \\
(\mathrm{m} / \mathrm{s})\end{array}$ & $\begin{array}{c}\text { Max. Froude } \\
\text { number } \\
(-)\end{array}$ \\
\hline 10 & 0.035 & 7.0 & 22.3 \\
\hline 20 & 0.05 & 5.0 & 11.3 \\
\hline 30 & 0.055 & 3.7 & 6.8 \\
\hline 40 & 0.06 & 3.1 & 4.9 \\
\hline 50 & 0.065 & 2.6 & 3.7 \\
\hline 60 & 0.07 & 2.3 & 3.0 \\
\hline
\end{tabular}

of the frame structure to the model base also prevented the transmission of vibrations from the glass channel to the measuring equipment and the occurrence of additional measurement uncertainty.

\section{Measuring equipment and measurements}

The main characteristics of standing waves along the junction for which phenomenological equations were developed, were obtained from a 3D mesh model of water surface topographies. Models of water surface topography were constructed from measurements with a laser scanner (Rak et al., 2017) (Fig. 2).

We used LIDAR instrument LMS400 manufactured by SICK AG. The device operates in the visible red light wavelength $\lambda=650 \mathrm{~nm}$. Its systematic measurement uncertainty is $\pm 4 \mathrm{~mm}$, while the statistical measurement uncertainty is \pm 3 $\mathrm{mm}$. The beam diameter is $1 \mathrm{~mm}$. For measurements we used configuration with a line scanning frequency $270 \mathrm{~Hz}$ and angular resolution $0.2^{\circ}$ (totalling 94500 distance measurements per second). For each scenario the water surface topography was measured over $2 \mathrm{~m}$ length with 22 cross sections and two centerlines per section (Fig. 3).

For each cross section the water surface profile was determined based on averaging measurements from 6,000 scan lines. Every scan line was composed of 350 measurement points, i.e. a total of 2,100,000 points in the entire point cloud of each cross section were recorded. A detailed description of measurement method, achieved measurement uncertainty and construction of water surface topography is given by Rak et al. (2017 and 2018). For referencing of measurements a local three-dimensional coordinate system was selected as shown in Fig. 3. The origin $(X=0, Y=0, Z=0)$ is located in point A.

\section{Description of experimental scenarios at the $T$-junction}

The independent adjustment of discharge and water depth of the incoming flow allowed for the settings of desired characteristics (flow depth, velocities and Froude number). Scenarios are classified into three groups. In the first group, Froude numbers of the incoming flows were between 2 and 12, while the water depths of incoming flows were set in $5 \mathrm{~mm}$ increments from 10 $\mathrm{mm}$ to $30 \mathrm{~mm}$. In each individual scenario the water depth of both incoming flows was identical. A total of 101 different scenarios were analysed in this group. In group 2, range of Froude number values was the same $(2 \leq \mathrm{Fr} \leq 12)$. We varied the incoming flow depth in the tributary channel $(10 \mathrm{~mm}, 20$ $\mathrm{mm}$ and $30 \mathrm{~mm}$ ) while the depth in the main channel remained constant at $30 \mathrm{~mm}$. A total of 53 different scenarios were analysed. In group 3 , we varied the incoming flow depth in the main channel $(10 \mathrm{~mm}, 20 \mathrm{~mm}$ and $30 \mathrm{~mm})$ while depth in the tributary channel remained constant at $30 \mathrm{~mm}$. Froude number of incoming flows of the junction were between 2 and 12. In group 3, a total of 57 different scenarios were analysed. 

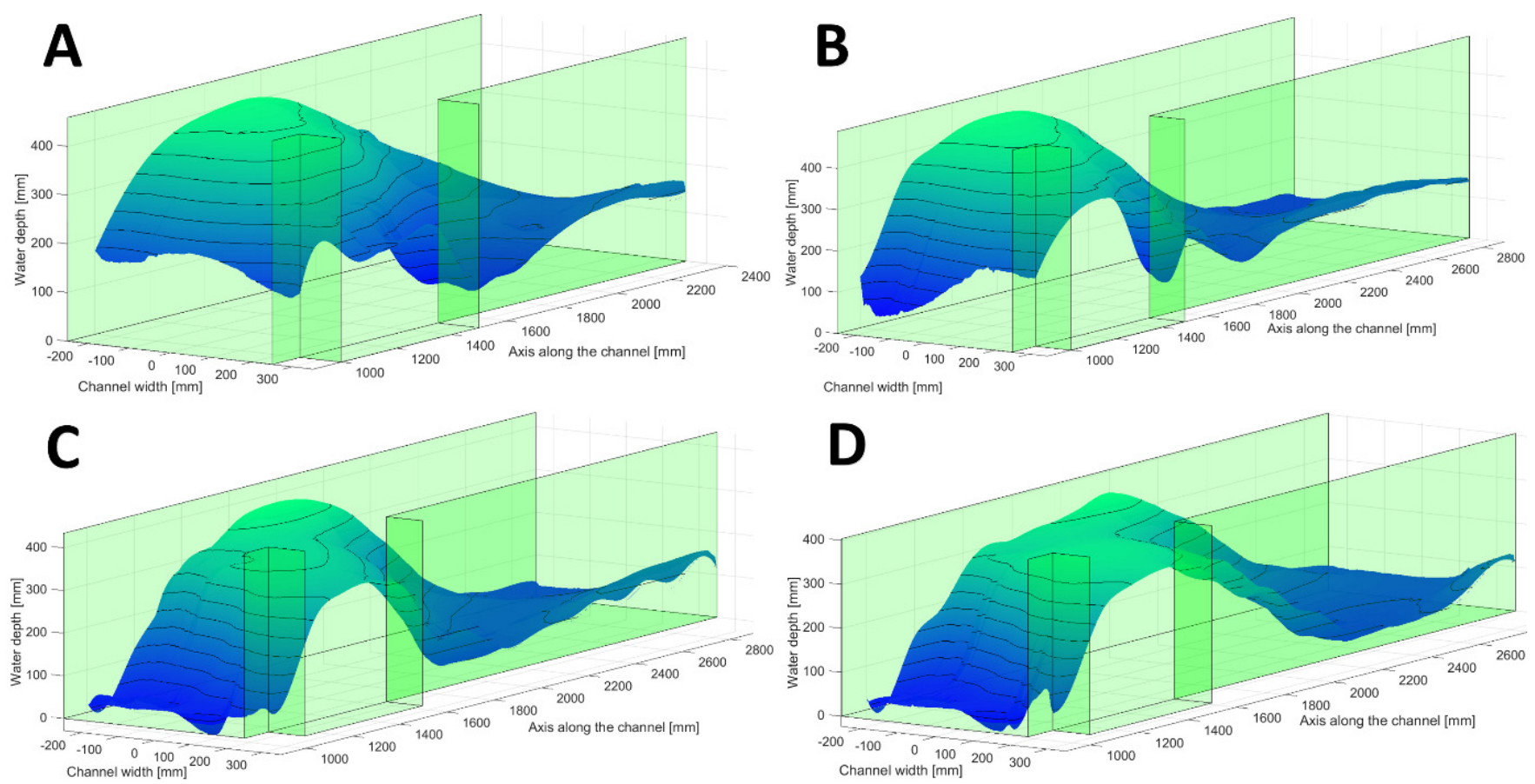

Fig. 2. Constructed water surface topographies along the junction using LIDAR data for 4 scenarios with different flow geometry and hydraulic conditions: A: $h_{m}=h_{t}=25 \mathrm{~mm}, \mathrm{Fr}_{m}=7.92$ and $\mathrm{Fr}_{t}=5.79 ; \mathrm{B}: h_{m}=30 \mathrm{~mm}, h_{t}=20 \mathrm{~mm}, \mathrm{Fr}_{m}=6.76$ and $\mathrm{Fr}_{t}=7.04 ; \mathrm{C}: h_{m}=30 \mathrm{~mm}$, $h_{t}=10 \mathrm{~mm}, \mathrm{Fr}_{m}=5.99$ and $\mathrm{Fr}_{t}=11.99 ; \mathrm{D}: h_{m}=30 \mathrm{~mm}, h_{t}=10 \mathrm{~mm}, \mathrm{Fr}_{m}=6.9$ and $\left.\mathrm{Fr}_{t}=11.05\right)$.

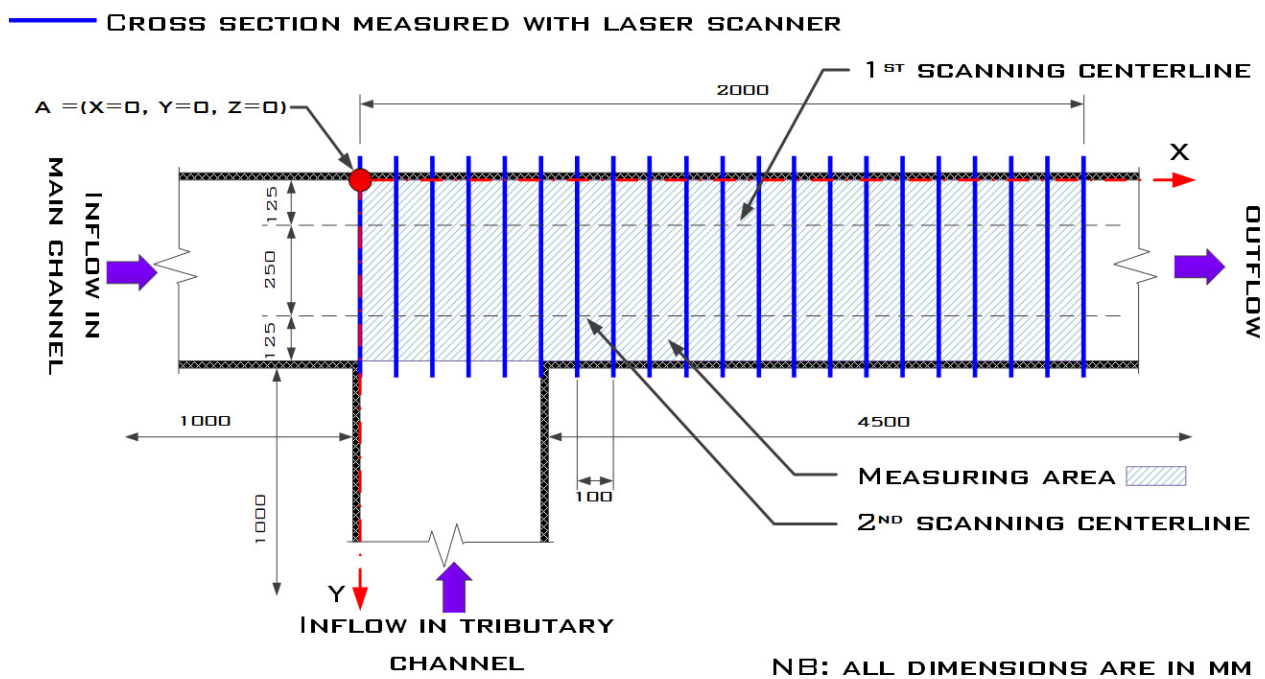

Fig. 3. Junction with lines of the measured cross sections.

For each scenario, we also control the flow conditions with values of Reynolds and Weber number. Reynolds number Re gives the ratio of inertial forces to the viscous forces within a fluid and is defined as $R e=\frac{\rho v l}{\mu}$. Values of Reynolds numbers were between $0.4 \times 10^{4}$ and $1.1 \times 10^{5}$. The Weber number $W e$ gives the ratio of inertial forces and forces due to surface tension. The Weber numbers were calculated using the following equation: $W e=\frac{v^{2} \rho l}{\kappa}$. To avoid the effect of forces due to surface tension on the flow the value of Weber number has be greater than 100 (Peakall and Warburton, 1996). In our scenarios values of the Weber number were between 120 and $6.2 \mathrm{x}$ $10^{3}$. The integrated table with input parameters and results for all scenarios can be found in the first author's doctoral dissertation (publicly available) (Rak, 2017).

\section{Development of equations with regression analysis}

To explore the forms of relationship between input data and characteristics of standing waves at the junction on the basis of experimental data, different regression models were considered, with the power-law approach proving to be the most appropriate. Due to the large set of measured scenarios, the system of nonlinear equations was overdetermined. To obtain a system of linear equations, we used a logarithm operation. To solve the system of equations and to determine the coefficients and exponents of power-law formulation, we used the least-squares method. Input parameters (geometric and hydraulic parameters, 
i.e. water depth, discharge of inflows, angle of junction) were used as predictors as well as some derived parameters (Fr numbers, reference lengths etc.). In the design of equations we verified the influence of each input and derived parameters on the characteristics of the standing waves. We defined 14 significant characteristics of standing waves (angle of mixing cone, peak heights, location of peaks etc.), for which simple design equations were developed with reasonable values of coefficients and exponents. Only the most influential parameters were included in the formulation, while the final equation was determined according to agreement between the measured values and the values calculated with the newly defined equation. The most appropriate formulation of the phenomenological equations was also verified by correlation analysis.

\section{RESULTS}

Although all the scenarios address hydraulic characteristics of the supercritical junction flow, diverse topographies of water surface occur. Four main types of flow patterns were observed at the junction. Among them cases with humped wave at the junction were considered as the most interesting and were selected for further analysis. Finally, a comparison of the present predictions with other researchers' results is provided.

\section{Water flow patterns}

The analysis of measurements of water surface topography in all scenarios showed that junction flow patterns do not only depend on Froude number but also on water velocities and water depths in the both approach branches. Therefore, a new quantity of ratio between momentums in main and tributary channels was introduced:

$$
M_{R}=\frac{M_{m}}{M_{m}+M_{t}}
$$

where $M_{m}$ and $M_{t}$ describe the rate of flow momentums in the main and tributary branches:

$$
\begin{aligned}
& M_{m}=v_{m}^{2} \cdot h_{m} \cdot b_{m} \cdot \rho_{w} \\
& M_{t}=v_{t}^{2} \cdot h_{t} \cdot b_{t} \cdot \rho_{w} .
\end{aligned}
$$

The channels width is constant $\left(b_{m}=b_{t}\right)$ and there is no multi-phase flow upstream of the junction, so $\rho_{w}=$ const .
On the basis of measurements and on the ratio between momentums of incoming flows the water flow patterns were classified into four main groups:

1) $\quad F r_{m} \leq 2 \rightarrow$ For Froude number below 2 the hydraulic jump or dammed flow occurs in both channels already upstream of the junction. Similar results were reported by Schwalt and Hager (1995); Hager (2010). Energy dissipation in the mixing zone of both incoming flows is greater at the T-shaped junction than at junctions of narrower angles.

2) $\quad M_{m} \leq M_{t} ; \mathrm{Fr}_{m, t}>2 \rightarrow$ For momentum in the tributary channel equal to or greater than momentum in the main channel, in both channels (between the outlet from the pressure vessels and the junction) hydraulic jump or submerged outflow from the pressure vessel is present. If submerged outflow is present the flow is dammed to a greater extent and hydraulic jump would occur at a greater distance upstream of the junction than the distance between the inlet and junction was in our experiment. In situations with much greater momentum of the incoming flow in the tributary channel than that in the main channel the hydraulic jump in the tributary channel occurs just before the junction. In junctions with a narrower angle it is possible that the supercritical flow of the tributary channel is preserved throughout the junction, while the flow in the main channel is dammed (Schwalt and Hager, 1995). This is not the case in the T-shaped junction (Fig. 4, left).

3) $M_{t} \leq M_{m} \leq 2.5 \cdot M_{t} ; F r_{m, t}>2 \rightarrow$ The momentums of the incoming flows in both channels are large enough to retain supercritical characteristics within the junction. Along a junction a standing humped wave occurs, while flows from main and tributary channel remain supercritical throughout the junction and also in the downstream channel. The height of the standing humped wave in the area of the junction is much greater than the potential water depth in the junction if hydraulic jump occurs in either of the channels upstream of the junction (Fig. 4, middle).

4) $\quad M_{m} \geq 2.5 \cdot M_{t} ; F r_{m}>2 \rightarrow$ The momentum of the incoming flow in the main channel is much greater than in the tributary channel, therefore choking of the flow occurs in the area of the junction within the main channel but supercritical conditions are preserved throughout the junction as well as further downstream of it. Disturbance of the flow caused by inflow from the tributary channel results in the mixing zone while the tributary flow is dammed (Fig. 4, right).
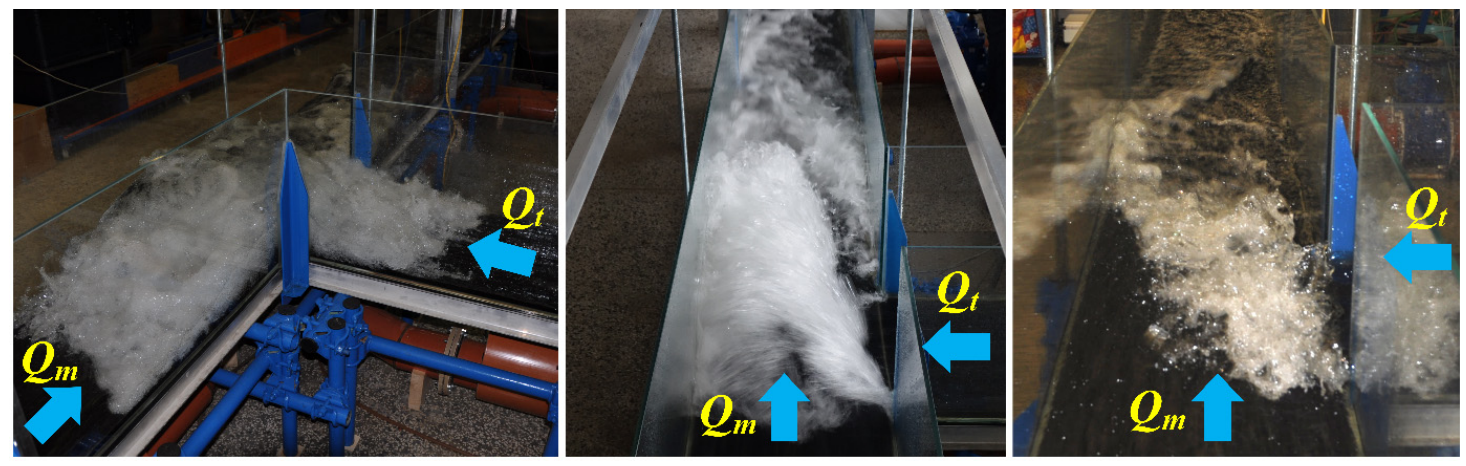

Fig. 4. Flow patterns in a junction: hydraulic jumps in both incoming channels $\left(h_{m}=h_{t}=25 \mathrm{~mm}, \mathrm{Fr}_{m}=8, \mathrm{Fr}_{t}=8\right)$ (left); humped wave $\left(h_{m}\right.$ $=h_{t}=20 \mathrm{~mm}, \mathrm{Fr}_{m}=8, \mathrm{Fr}_{t}=6$ ) (middle); choking of the main channel flow, while the side incoming flow is dammed $\left(h_{m}=h_{t}=20 \mathrm{~mm}, \mathrm{Fr}_{m}\right.$ $\left.=8, \mathrm{Fr}_{t}=2\right)($ right). 


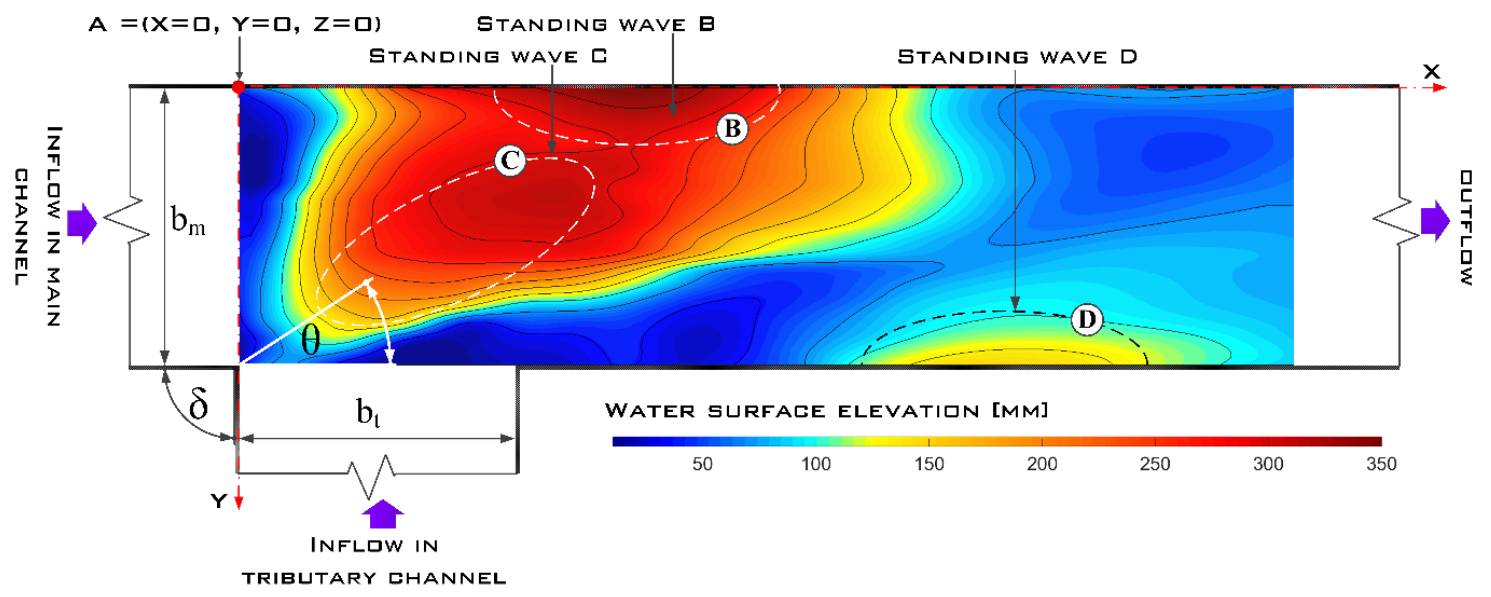

Fig. 5. The main flow patterns of water flow for which parametric relations with input parameters are given.

\section{Topology of water surface of standing waves}

Although depths of the incoming flows in both channels upstream of the junction are low, a humped wave is formed over the junction due to high velocities of the incoming flows. The humped wave is characterised by high velocity and complex, non-stationary topography of the water surface. Due to the disturbance and turbulence of the water flow in the mixing zone the air entrainment and the two-phased flow occurs. In the case of humped wave, the water surface was found to be more than ten times higher than the depth of incoming flows to the junction while flow over the junction remains supercritical. The measurements show that intensive vertical dynamics translates to about $80 \%$ of energy dissipation of the incoming flows but nonetheless the flow retains in the supercritical regime throughout (downstream of the junction as well). Characteristics of standing waves, which were considered in phenomenological analysis are presented in Fig. 5 with letters C, B and D, following the definition of Schwalt and Hager (1995). Standing wave $\mathrm{C}$ is formed along the mixing zone of both incoming flows. Due to the incoming flow from the tributary channel streamlines in the main channel are deflected toward the lateral wall, where wave B occurs. Reflection of wave B leads to wave $\mathrm{D}$ along the wall on the opposite side.

The main characteristics of standing waves are defined with longitudinal coordinate $(X)$, transverse coordinate $(Y)$ and height of the wave peaks $(Z)$. The origin of the coordinate system is at the beginning of the junction (longitudinal axis), at the lateral wall of the main channel (transverse axis), and at the bottom of the channel (vertical axis) (Fig. 3 and 5).

The range of validity of equations is set based on our selection of scenarios. Equations are in the valid range when the

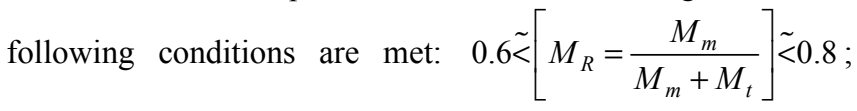
$F r_{m}>6 ; F r_{t}>3 ; b=b_{m}=b_{t}$ and $\delta=90^{\circ}$

Equations give dimensions and location of standing waves. To set the peak height of the wave crest both equation of mean water surface level and equation of fluctuation of water surface are considered. Notations are specified at the end of this article.

\section{Results of parametric modelling}

In the following results of parametric modelling of standing wave properties at the junction are provided.
The angle of mixing zone $\theta$ or crest of wave $\mathrm{C}$ is set as:

$$
\theta=\tan ^{-1}\left(\frac{b-y_{M C}}{x_{M C}}\right)
$$

where $\tan \theta$ was calculated using parametric analysis:

$$
\tan \theta=\left(\frac{b-y_{M C}}{x_{M C}}\right)=0.9 \cdot\left(\frac{h_{t}}{h_{m}}\right)^{1.2} \cdot f^{0.8}
$$

Main characteristics of the wave $C$

- Longitudinal coordinate of the peak of wave C

$X_{M C}=\frac{x_{M C}}{b}=1.1 \cdot(\cos \theta)^{(-4.5)} \cdot\left(\frac{h_{t}}{h_{m}}\right)^{(-0.6)} \cdot F r_{m}^{0.8} \cdot F r_{t}^{0.1} \cdot\left(\frac{b}{\bar{h}}\right)^{(-0.85)}$

- Transverse coordinate of the peak of wave C

$Y_{M C}=\frac{y_{M C}}{\bar{h}}=b-2 \cdot(\sin \theta)^{(-1.5)} \cdot\left(\frac{h_{t}}{h_{m}}\right)^{3.7} \cdot F r_{m}^{(-2.5)} \cdot F r_{t}^{3.7}$

- $\quad$ The peak height of wave $\mathrm{C}$ (mean value)

$Z_{M C}=\frac{h_{M C}}{\bar{h}}=F r_{m} \cdot F r_{t}^{1.4} \cdot\left(\frac{b}{\bar{h}}\right)^{(-0.6)}$

- Free-water surface fluctuations at the peak of wave C

$H_{M C}^{\prime}=\frac{h_{M C}^{\prime}}{h}=0.11 \cdot F r_{m}^{1.1} \cdot F r_{t}^{0.2}$

- Actual height of the peak of wave C

$Z_{M C, a c t}=Z_{M C} \pm H_{M C}^{\prime}$

Main characteristics of wave B (at the wall opposite the inflow of the tributary channel)

- Longitudinal coordinate of the peak of wave B

$X_{M B}=\frac{x_{M B}}{b}=(\cos \theta)^{4} \cdot F r_{m}^{0.25} \cdot F r_{t}^{0.15}$ 
- $\quad$ The peak height of wave B (mean value)

$Z_{M B}=\frac{h_{M B}}{\bar{h}}=1.1 \cdot F r_{m}^{0.9} \cdot F r_{t}^{1.5} \cdot\left(\frac{b}{\bar{h}}\right)^{(-0.6)}-1$

- $\quad$ Free-water surface fluctuations at the peak of wave B

$H_{M B}^{\prime}=\frac{h_{M B}^{\prime}}{\bar{h}}=0.16 \cdot F r_{m}^{0.6} \cdot F r_{t}^{0.75}$

- $\quad$ Actual height at the peak of wave B

$Z_{M C, a c t}=Z_{M B} \pm H_{M B}^{\prime}$

- Longitudinal coordinate of downstream end of the crest of wave B

$$
X_{E B}=\frac{x_{E B}}{\bar{h}}=0.75 \cdot\left(\frac{h_{t}}{h_{m}}\right)^{(-1.3)} \cdot F r_{m}^{1.5} \cdot F r_{t}^{(-0.9)} \cdot\left(\frac{b}{\bar{h}}\right)^{0.75}+\frac{b}{2 \bar{h}}
$$

Main characteristics of the wave D (reflection of wave $B$ - along the wall on side of the tributary channel)

- Longitudinal coordinate of the peak of wave D

$X_{M D}=\frac{x_{M D}}{\bar{b}}=1+0.45 \cdot\left(\frac{h_{t}}{h_{m}}\right)^{(-2.1)} F r_{m}^{2.3} \cdot F r_{t}^{(-1.2)} \cdot\left(\frac{b}{\bar{h}}\right)^{(-0.5)}$

- $\quad$ The peak height of wave D (mean value)

$Z_{M D}=\frac{h_{M D}}{\bar{h}}=2.3 \cdot F r_{m}^{0.7} \cdot F r_{t}^{0.8} \cdot\left(\frac{b}{\bar{h}}\right)^{(-0.55)}$.

- $\quad$ Free-water surface fluctuations at the peak of wave D

$H_{M D}^{\prime}=\frac{h_{M D}^{\prime}}{\bar{h}}=0.1 \cdot F r_{m}^{\frac{2}{3}} \cdot F r_{t}^{\frac{2}{3}}$

- Actual height at the peak of wave D

$Z_{M D, a c t}=Z_{M D} \pm H_{M D}^{\prime}$

Correlation between measured values in the model and calculated values with the newly proposed phenomenological equations is high despite nonstationariness and complexity of the phenomenon. Correlation factor is mainly $\mathrm{R}^{2} \geq 0.9$. Values of the correlation factors $\mathrm{R}^{2}$ for each equation are given in Table 2 . There is deviation in certain points but they still fall within $\pm 10 \%$ confidence interval. A lower correlation coefficient $\left(\mathrm{R}^{2} \sim 0.6-0.7\right)$ is achieved for fluctuating.

According to previous research, a modelling of high-speed two-phase flow without the scale effect would be possible, when Froude number, Weber number and Reynolds number are identical in the laboratory model and the larger model or prototype. Physically, it is only possible when a full-scale model is used (Murzyn and Chanson, 2008; Pfister and Chanson, 2014). Therefore, our results could not be simply extrapolated to a larger scale, based upon a Froude similarity. To do this, it would be appropriate to extend our research with the set of additional experimental set-ups to enable scale-up investigations. To minimise the scale effect some condition has to considered (e.g. Reynold number, Weber number or Morton number).
Table 2. Values of the correlation factors for all equations.

\begin{tabular}{|l|c|}
\hline \multicolumn{1}{|c|}{ Characteristics of standing waves } & $\mathrm{R}^{2}$ \\
\hline Angle of mixing zone & 0.97 \\
\hline Longitudinal coordinate of the peak of wave C & 0.93 \\
\hline Transverse coordinate of the peak of of wave C & 0.95 \\
\hline The peak height of wave C & 0.96 \\
\hline Free-water surface fluctuations at the peak of wave C & 0.66 \\
\hline Longitudinal coordinate of the peak of wave B & 0.94 \\
\hline The peak height of wave B & 0.89 \\
\hline Free-water surface fluctuations at the peak of wave B end of the crest of & 0.80 \\
\hline $\begin{array}{l}\text { Longitudinal coordinate of downstream end } \\
\text { wave B }\end{array}$ & 0.84 \\
\hline Longitudinal coordinate of the peak of wave D & 0.93 \\
\hline The peak height of wave D & 0.93 \\
\hline Free-water surface fluctuations at the peak of wave D & 0.62 \\
\hline
\end{tabular}

\section{Comparison with Schwalt-Hager results}

The results of the present experiments were compared with the findings of Schwalt and Hager (1995), who experimentally set equations for the characteristics of standing waves in junctions in a similar way as in our work. Their research included experiments with junction angles up to $60^{\circ}$, so they limited the range of validity of their equations with angles of junction up to $70^{\circ}$. Since it is the only experiment comparable to ours, we did a side by side comparison of characteristics of standing waves that were included in both experiments despite the disagreement in valid junction angles. On the other hand, Hager (2010) stated that at the right-angled junction the backwater effect always occurs in both inflow channels. Our experiments prove otherwise-humped wave (a water surface pattern that was formed in the Schwalt-Hager experiment) also forms with a right-angled junction at certain conditions.

Some equations of Schwalt-Hager (1995) contain $\cos \delta$ as a denominator, so their results were not directly comparable since in our case $\cos \delta=0$ for $\delta=90^{\circ}$. These are, for example, longitudinal coordinate of the peak height of wave $\mathrm{C}$ and distance of wave end from the junction for wave B. According to our experiments, the characteristics of standing waves depend on the angle of the mixing zone or wave $\mathrm{C}$ (the latter being dependent on the momentum of both main and tributary channel flows). Fig. 6 shows that the Schwalt-Hager (1995) equations underestimate the angle of the mixing zone in comparison with our results. Similarly, the equations of Schwalt and Hager (1995) also underestimate other topographic characteristics of standing waves at the junction with the exception of equations for longitudinal coordinates of standing wave peaks, which overestimate the values. This disagreement is to be expected - in junctions with narrower angle momentum is directed along the channel downstream of the junction. Therefore, standing waves were also formed further in the downstream direction. Contrary, vertical dynamics and the height of standing waves, as well as the dissipation of energy (due to transverse dynamic of water mass) are much bigger in the junction with a $90^{\circ}$ angle. These properties also lead to concentration of the phenomena in the area of the junction.

Due to the use of classical measuring instruments the Schwalt-Hager research did not analyse water surface fluctuations of standing waves. Although other authors (Chachereau and Chanson, 2010; Murzyn and Chanson, 2009) did set fluctuations of water surface at the hydraulic jump in the channel, direct comparison with fluctuations in our experiment is not possible due to the significantly different dynamics of the water surface at the junction. However, both research studies show that fluctuations of the water surface of turbulent flow depend on the hydraulic characteristics of the incoming flow(s). 


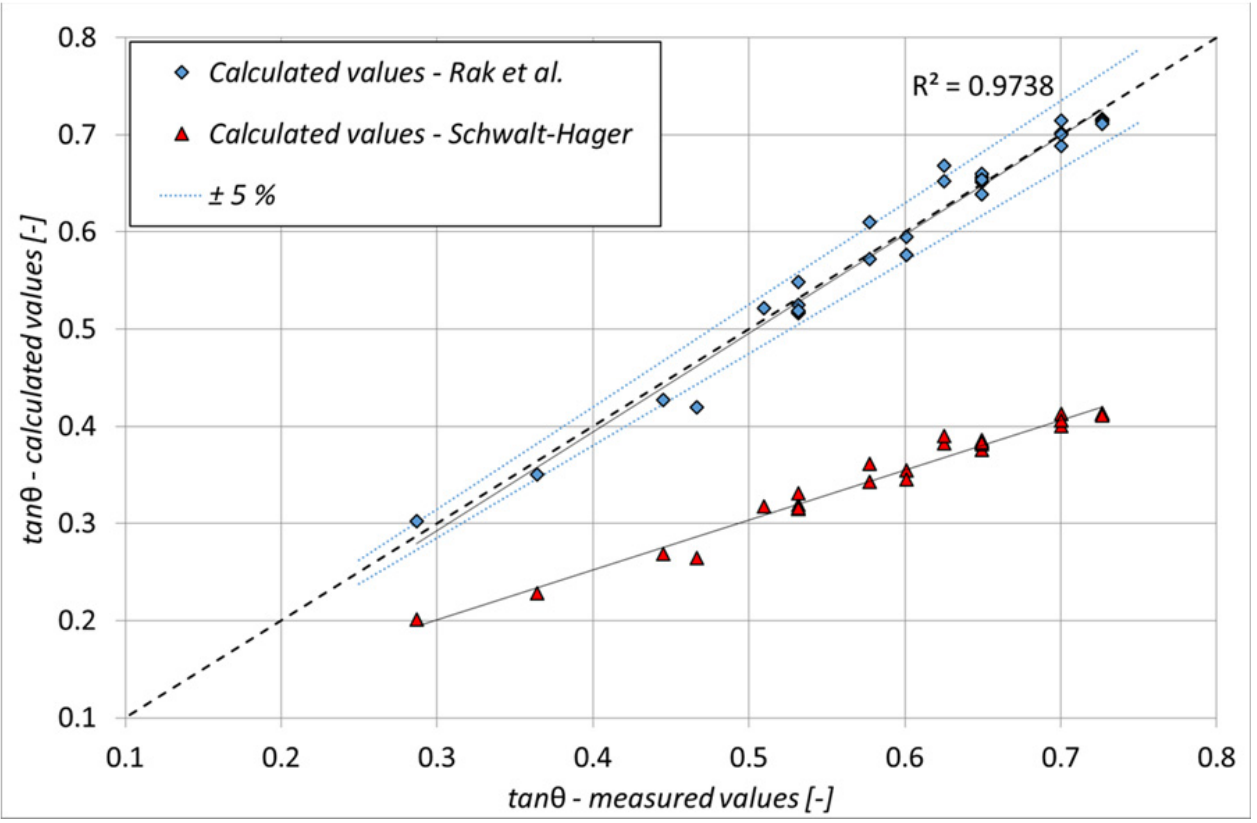

Fig. 6. Comparison between the values calculated with new equations determined in our study and Schwalt-Hager equations, and measurements in the experiment of the T-junction.

\section{CONCLUSION}

The topology of the water surface at a right-angled junction subjected to supercritical incoming flows is described. In an extensive experimental research study with non-intrusive measurement method, the topographies of the water surface at the junction were systematically measured. On the basis of analysis of the flow characteristics in the area of the junction the water flow patterns were classified by the parameters of the incoming flows, followed by a detailed analysis of characteristics of humped-shaped standing waves forming at the junction. Based on the measured water surface topography of the individual scenario where the humped wave occurred, the main characteristics of standing waves were determined. Dimensions of standing waves and their locations were used in a topological analysis and for development of dimensionless phenomenological equations providing the relations between input geometrical and hydraulic parameters, and the characteristics of the distinct standing wave patterns in the junction area. Despite the strongly developed turbulent two-phase flow with high water surface dynamics in the junction area, the correlation analysis revealed a very good fit across the entire range of the measured values in many scenarios. Knowing the characteristics of the phenomenon where supercritical flow is preserved across the junction, while high wave peaks occur along the junction that highly exceed incoming flow depths as well as knowing the relations between input parameters and wave characteristics brings important new knowledge in water engineering.

\section{REFERENCES}

Behlke, C.E., Pritchett, H.D., 1966. The design of supercritical channel junction. Highway Research Record, 133, 17-35.

Best, J., Reid, I., 1984. Separation zone at open channel junction. J. Hydraul. Eng., 110, 11, 1588-1594.

Bowers, C.E., 1950. Studies of open channel junctions. Project Report 24. Part V. Hydraulic model studies for Whiting Field Naval Air Station. University of Minnesota, St. Anthony Falls Hydraulic Laboratory, Minneapolis.
Chachereau, Y., Chanson, H., 2010. Free-surface fluctuations and turbulence in hydraulic jumps. Exp. Therm. Fluid Sci., $35,6,896-909$.

Christodoulou, G.C., 1993. Incipient hydraulic jump at channel junction. J. Hydraul. Eng., 119, 3, 409-423.

Greated, C.A., 1968. Supercritical flow through junctions. La Houille Blanche, 23, 3, 693-695.

Hager, W.H., 1989a. Supercritical Flow in Channel Junctions. J. Hydraul. Eng., 115, 5, 595-616.

Hager, W.H., 1989b. Transitional Flow in Channel Junctions. J. Hydraul. Eng., 115, 2, 243-259.

Hager, W.H., 2010. Wastewater Hydraulics: Theory and Practice. 2nd Edition. Springer Verlag, Heidelberg, Berlin.

Mignot, E., Riviere, N., Perkins, R., Paquier, A., 2008. Flow patterns in a four-branch junction with supercritical flow. J. Hydraul. Eng., 134, 6, 701-713.

Murzyn, F., Chanson, H., 2008. Experimental assessment of scale effects affecting two-phase flow properties in hydraulic jumps. Exp. Fluids., 45, 3, 513-521.

Murzyn, F., Chanson, H., 2009. Free-surface fluctuations in hydraulic jumps: Experimental observations. Exp. Therm. Fluid Sci., 33, 7, 1055-1064.

Peakall, J., Warburton, J., 1996. Surface tension in small hydraulic river models - the significance of the Weber number. J. Hydrol., 35, 2, 199-212.

Pfister, M., Chanson, H., 2014. Two-phase air-water flows: Scale effects in physical modelling. J. Hydrodyn. Ser. B, 26, 2, 291-298.

Pfister, M., Gisonni, C., 2014. Head losses in junction manholes for free surface flows in circular conduits. J. Hydraul. Eng., 140, 9, 1-6.

Pinto Coelho, M.M., 2015. Experimental determination of free surface levels at open-channel junction. J. Hydraul. Res., 53, 3, 394-399.

Rak, G., 2017. Topološka struktura vodne gladine na sotočju pri deročem toku (Water surface topology of supercritical confluence flow). Doctoral dissertation, University of Ljubljana, Faculty of Civil and Geodetic Engineering, 112 p. (in Slovene, with English captions and extended summary). 
https://repozitorij.uni-lj.si/ IzpisGradiva.php?id= 99202 \&lang=eng

Rak, G., Steinman, F., Hočevar, M., 2017. Measuring water surface topography using laser scanning. Flow Meas. Instrum., 56, 35-44.

Rak, G., Hočevar, M., Steinman, F., 2018. Construction of water surface topography using LIDAR data. Stroj. Vestn.-J. Mech. E., 64, 1-11.

Saldarriaga, J., Rincon, G., Moscote, G., Trujillo, M., 2017. Symmetric junction manholes under supercritical flow conditions. J. Hydraul. Res., 53, 3, 135-142.

Schwalt, M., Hager, W.H., 1995. Experiments to supercritical junction flow. Exp. Fluids, 18, 429-437.

Received 1 February 2018 Accepted 7 March 2018

\section{NOMENCLATURE \\ Subscripts:}

$C=$ standing wave in the mixing zone (humped wave)

$B=$ standing wave at the wall opposite tributary inflow

$D$ = standing wave at the side of tributary inflow (reflection of wave B)

$m \quad$ = main channel

$t \quad=$ tributary (side) channel

$M \quad$ = maximum value (wave height)

$E \quad=$ downstream end of standing wave

\section{Symbols:}

$X \quad=$ axis along the main channel

$Y=$ axis perpendicular to the main channel (aligned with tributary channel)

$Z \quad=$ vertical axis

$A(X=0, Y=0, Z=0)=$ origin of coordinate system (start of junction, left edge of the main channel (opposite the tributary channel), bottom of the channel)
$M_{R}=$ quantity of ratio between momentums in main and tributary channels $(-)$

$M_{m}=$ momentum of the inflow in the main channel

$M_{t} \quad=$ momentum of the inflow in the tributary channel

$\delta \quad=$ angle of junction (in our experiment $\delta=90^{\circ}$ )

$\theta \quad=$ angle of mixing zone (such as line of crest at the contact of two supercritical flows)

$h_{m} \quad=$ water depth of inflow in the main channel (m)

$h_{t} \quad=$ water depth of inflow in the tributary channel (m)

$v \quad=$ velocity of water flow $(\mathrm{m} / \mathrm{s})$

$v_{m}=$ velocity of inflow in the main channel $(\mathrm{m} / \mathrm{s})$

$v_{t} \quad=$ velocity of inflow in the tributary channel $(\mathrm{m} / \mathrm{s})$

$H^{\prime} \quad=$ dimensionless form of free surface fluctuation height $(-)$

$h^{\prime} \quad=$ fluctuation height $(\mathrm{m})$

$b=b_{m}=b_{t}=$ width of all channels (constant in our experiment) (m)

$\bar{b}=\left(b_{m} \cdot b_{t}\right)^{0.5}=$ ratio of width of both channels (m)

$l=$ characteristic length $(\mathrm{m})$

$\rho \quad=$ water density $\left(\mathrm{kg} \cdot \mathrm{m}^{-3}\right)$

$\mu \quad=$ dynamic viscosity $\left(\mathrm{kg} \cdot \mathrm{m}^{-1} \cdot \mathrm{s}^{-1}\right)$

$g \quad=$ gravitational acceleration $\left(\mathrm{m} / \mathrm{s}^{2}\right)$

$\kappa=$ surface tension $\left(\mathrm{N} \cdot \mathrm{m}^{-2}\right)$

\section{Derivatives:}

$\bar{h}=\left(h_{m} \cdot h_{t}\right)^{0.5}=$ characteristic water depth at the junction, calculated by characteristics of both inflows $(\mathrm{m})$

$F r_{m}=\frac{v_{m}}{\sqrt{\left(\mathrm{g} \cdot h_{m}\right)}}=$ Froude number of inflow in the main channel $(-)$

$F r_{t}=\frac{v_{t}}{\sqrt{\left(\mathrm{g} \cdot h_{t}\right)}}=$ Froude number of inflow in the tributary channel (-)

$f=\frac{F r_{m} \cdot F r_{t}}{\left(F r_{m}+F r_{t}\right)}=$ ratio of Froude numbers of both inflows (-) 